\title{
PATTERN FORMATION ON THE PROTOTYPE COMPLEX-CELL CNN-UM CHIP (CACE1K)
}

\author{
Dávid Bálya, István Petrás and Csaba Rekeczky \\ AnaLogic and Neural Computing Systems Laboratory \\ Computer and Automation Institute of the Hungarian Academy of Sciences \\ 1111 Budapest, Kende u. 13-17, Hungary
}

\begin{abstract}
In this paper several pattern formation phenomena generated on a prototype complex cell (second order) CNN-UM chip (CACE1k) will be demonstrated. It is shown that the basic motifs (patches, checkers and stripes) can be easily calculated and furthermore, the interaction of the two dynamic layers leads to new patterns that cannot be generated on a first order architecture. Throughout the paper a rich set of measured phenomena is given motivating further research toward a constructive use of pattern formation dynamics in various flow processing algorithms.
\end{abstract}

\section{INTRODUCTION}

In recent works we have reported several wave-type [1] and retinotopic processing [2] phenomena on a prototype complex cell CNN-UM chip (CACE1k, [3]). While these experiments showed a remarkable complexity of easily programmable functionality they have explored only a relatively small region of the available parameter space. In the continuation of these efforts now we have been focusing on various pattern generation possibilities and, for the first time, demonstrate a very rich set of results measured on the CACE1k chip (all the presented images were generated by this chip). Since all of these outputs are generated by a single instruction cooperative-competitive computation the examples also contribute to the set of operations frequently referred as having "single transient" dynamics. Moreover these configurations are not like traditional reaction-diffusion networks, since the intralayer couplings could also be inhibitory.

We do believe that apart from their fundamental relevance to many phenomena observed in nature the constructive use of these operators in signal/image processing algorithms is still a very challenging research topic. It is our hope that these measurement results of this paper demonstrate how the basic patterns can be generated on a silicon chip in the time range of a few microseconds, and the easy way of programming to make all kinds of interactions will motivate further intensive research on this area.

\section{THE CACE1K CNN-UM CHIP}

The architecture of the CACE1k chip is similar to the standard one layer CNN-UM [4] chip (ACE4k [5]), but its first order cell core is replaced by a second order one (see in Fig.1).

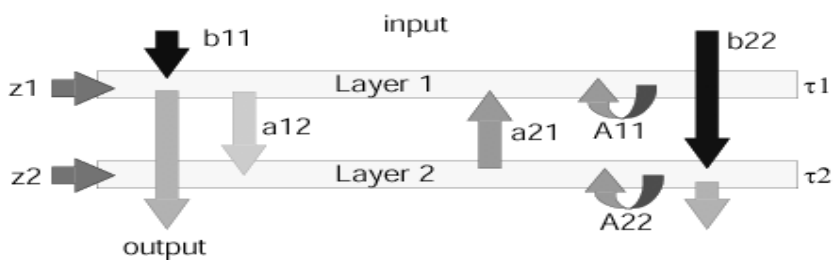

Fig.1. The processing structure of the complex-cell cellular neural network chip: CACE1k. Horizontal bars represent 2-dimensional locally coupled cell arrays.

The canonical state equation describing the dynamics of the complex cell unit is as follows (based on the socalled full-signal range model):

$$
\begin{array}{ll}
\text { Layer1 } & \tau_{1} \dot{x}_{1, i j}=-g\left(x_{1, i j}\right)+\sum_{k l \in N_{1}} A_{11, k l} x_{1, k l}+a_{21} x_{2, i j}+b_{11} u_{1, i j}+z_{1, i j} \\
\text { Layer2 } & \tau_{2} \dot{x}_{2, i j}=-g\left(x_{2, i j}\right)+\sum_{k l \in N_{1}} A_{22, k l} x_{2, k l}+a_{12} x_{1, i j}+b_{22} u_{2, i j}+z_{2}, i j
\end{array}
$$
where the nonlinearity $g($.) can be described as:

$$
g(x)=\lim _{m \rightarrow \infty}\left\{\begin{array}{cc}
m(x-1) & \text { if } x>1 \\
x & \text { if }|x| \leq 1 \\
m(x+1) & \text { if } x<-1
\end{array}\right.
$$

The dynamic evolution law of the complex cell CNN is described by the above system of differential equations and can be explained as follows. Each CNN node receives contributions from the processing nodes in the nearest neighborhood, which are summed and integrated in the state capacitor. The time constants of the two layers are different. The first layer has a scalable time constant, 
controlled by the appropriate binary code ( $\alpha \tau_{\mathrm{CNN}}$, where $\alpha$ is an integer between 1 and 16), while the second layer has a fixed time constant $\left(\tau_{\mathrm{CNN}}=100 \mathrm{~ns}\right)$. The evolution of the state variable $(x)$ is also determined by a self-feedback and by the feed-forward action of the stored input $(u)$ and bias ( $z$ ) values. There is a voltage limiter $(g)$ for implementing the full signal range (FSR) property of the implemented CNN-UM resulting in the very same state variable that is the output of the system [6].

The prototype chip has been designed and fabricated in a $0.5 \mu \mathrm{m}$ single-poly triple-metal CMOS technology. Its dimensions are $9.27 \times 8.45 \mathrm{~mm}^{2}$, which contain 32 by 32 complex-cells. The programmable dynamics of the chip permit the observation of different phenomena of the complex wave-instructions and pattern formations.

The CACE1k chip is integrated into a professional development environment, called Aladdin Professional [7]. The software environment supports several different methods to reach the potential of the chip from a high level language to low level direct commands.

\section{CONFIGURATION}

The paper presents the most interesting results from the different pattern-formation chip experiments carried out on the CACE1k cellular visual microprocessor. In the pattern formation measurements, a two-layer autonomous CNN structure was used. Thus, the parameters have the following structure:

$$
A_{11}=\left[\begin{array}{ccc}
r_{1} & s_{1} & r_{1} \\
s_{1} & p_{1} & s_{1} \\
r_{1} & s_{1} & r_{1}
\end{array}\right], a_{21}, \tau ; \quad A_{22}=\left[\begin{array}{ccc}
r_{2} & s_{2} & r_{2} \\
s_{2} & p_{2} & s_{2} \\
r_{2} & s_{2} & r_{2}
\end{array}\right], a_{12}
$$

The $\tau$ is the relative time constant of the first layer. According to the chip experiments, usually this value does not qualitatively influence the steady state/equilibrium points of the system, only the trajectory is changed. Both layers' states were initialized with similar random images, shown in Fig.2. The results/final patterns were read out as binary images after the steady state, decided based on visual inspection, was reached (or maximum $4000 \tau_{\mathrm{CNN}}$ was elapsed).

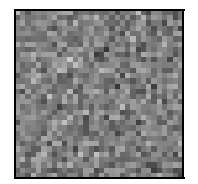

Fig.2. The random initial state for both layers

\section{EXPERIMENTS}

The three basic types of patterns that can be generated on single-layer architecture using nearest neighbor linear templates are patches, checkers, and stripes (Thiran et. al [8]). All the basic three patterns can be generated on both layers of the CACE1k chip (see examples for the first layer in Fig. 3). The template parameters for the chip are given below each characteristic pattern. The theoretical parameter region for a given pattern is also known [9].

\begin{tabular}{|c|c|c|}
\hline Patch & Checker & Stripe \\
\hline & 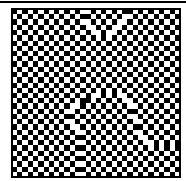 & 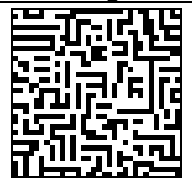 \\
\hline $\mathrm{r}=0.5 \mathrm{~s}=1 \mathrm{p}=2$ & $\mathrm{r}=0 \mathrm{~s}=-1 \mathrm{p}=0$ & $\mathrm{r}=-1 \mathrm{~s}=0 \mathrm{p}=1$ \\
\hline$p+4 s+4 r>0$ & $p-4 s+4 r>0$ & $\mathrm{p}-4 \mathrm{r}>0$ \\
\hline
\end{tabular}

Fig.3. The three basic patterns and their rules

The 2-layer structure extends the possibilities by combining two different pattern formation dynamics. The combinations of the basic patterns are shown for a given layer interaction in Fig.4. In these experiments the pattern formed on the second layer (L2) is negatively coupled into the first layer (L1). The interacting pattern on the first layer is displayed. The pattern generating parameters are shown in Fig.3.

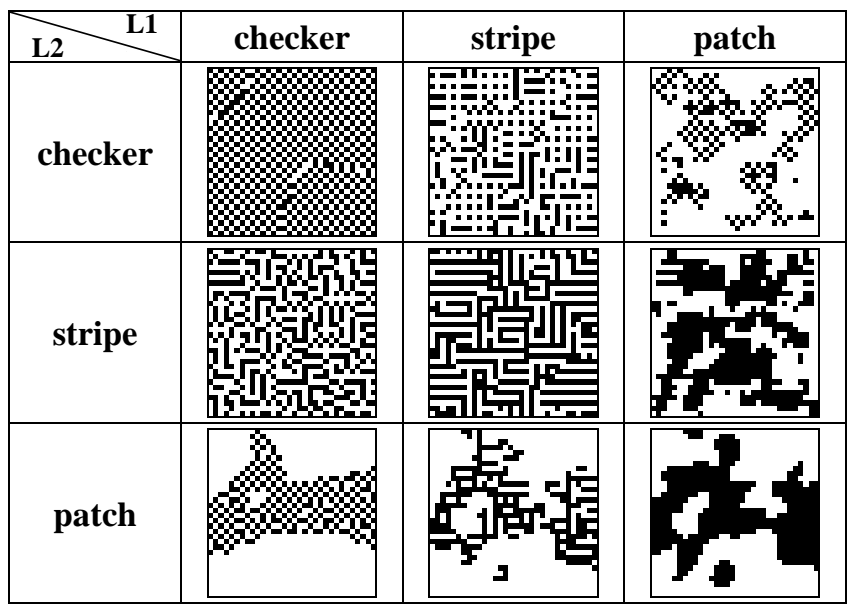

Fig.4. Interacting patterns $\left(a_{21}<0, a_{12}=0, L 1\right.$ 's output)

Reverting the sign of the interlayer couplings in case of two coupled checkerboard patterns synchronization phenomena has been found. It has also been observed that positive coupling results the same- (Fig.5a), while the negative anti-phase (Fig.5b) patterns. Although, this is not entirely true for the boundary regions where a number of discrepancies can be found (see Fig.5). 


\begin{tabular}{|c|c|}
\hline Parameters & Generated patterns (L1 L2) \\
\hline $\begin{array}{l}r_{1}=0 s_{1}=-1 p_{1}=0 \\
r_{2}=0 s_{2}=-1 p_{2}=0 \\
a_{12}=0 \mathbf{a}_{21}=\mathbf{1}\end{array}$ & 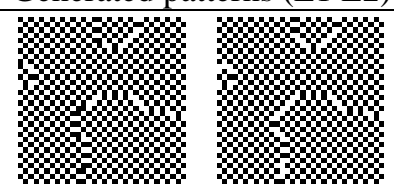 \\
\hline $\begin{array}{l}r_{1}=0 s_{1}=-1 p_{1}=0 \\
r_{2}=0 s_{2}=-1 p_{2}=0 \\
a_{12}=0 a_{21}=-\mathbf{1}\end{array}$ & 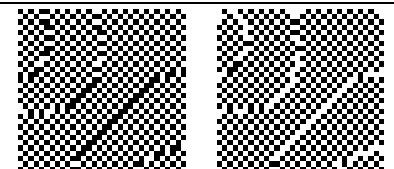 \\
\hline
\end{tabular}

Fig.5. Interacting checkerboard patterns ( $\mathrm{a}_{21}$ is changed)

In the next set of experiments it has been tested how qualitatively different patterns influence each other during the evolution.

Several parameter settings are measured and we have found that generating a pattern in one layer and coupling to the other can:

- $\quad$ significantly modify the steady-state properties (a, b);

- $\quad$ act as a region selector between two different patterns appearing on the layer (c);

- mask the other layer and enable/disable pattern formation in the patch region ( $g, i, j)$;

- $\quad$ insert its pattern into the existing pattern (e, h);

- $\quad$ modulate the patterned region (d);

- $\quad$ or generally interact (f, k).

Fig. 6 summarizes some interesting cases, without the completeness of the pattern interaction universe. In the table those specific outputs (c, d) are also shown where the basic motifs appear embedded into one another, as it was predicted in the early phase of the complex cell architecture design and simulation experiments [10]. For the chip experiments the templates and the output of both layers are given in the table. The parameter $\tau$ has no significant role in these cases.

\begin{tabular}{|c|c|c|}
\hline Parameters & Generated patterns (L1 L2) & \\
\hline $\begin{array}{l}\mathrm{r}_{1}=-0.5 \mathrm{~s}_{1}=0 \mathrm{p}_{1}=2 \\
\mathrm{r}_{2}=0.5 \mathrm{~s}_{2}=1 \mathrm{p}_{2}=2 \\
\mathrm{a}_{12}=0 \mathrm{a}_{21}=0.5\end{array}$ & 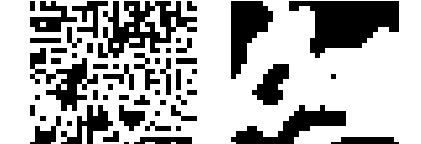 & $\mathrm{a}$ \\
\hline $\begin{array}{l}\mathrm{r}_{1}=0.5 \mathrm{~s}_{1}=0 \mathrm{p}_{1}=2 \\
\mathrm{r}_{2}=-1 \mathrm{~s}_{2}=0 \mathrm{p}_{2}=2 \\
\mathrm{a}_{12}=0 \mathrm{a}_{21}=0.9\end{array}$ & 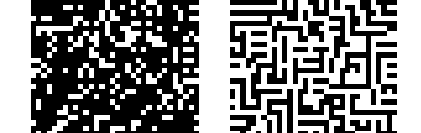 & $\mathrm{b}$ \\
\hline $\begin{array}{l}\mathrm{r}_{1}=-0.5 \mathrm{~s}_{1}=-1 \mathrm{p}_{1}=0 \\
\mathrm{r}_{2}=0.5 \mathrm{~s}_{2}=1 \mathrm{p}_{2}=2 \\
\mathrm{a}_{12}=0 \mathrm{a}_{21}=1\end{array}$ & 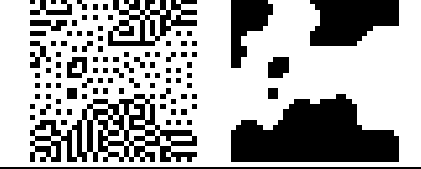 & C \\
\hline
\end{tabular}

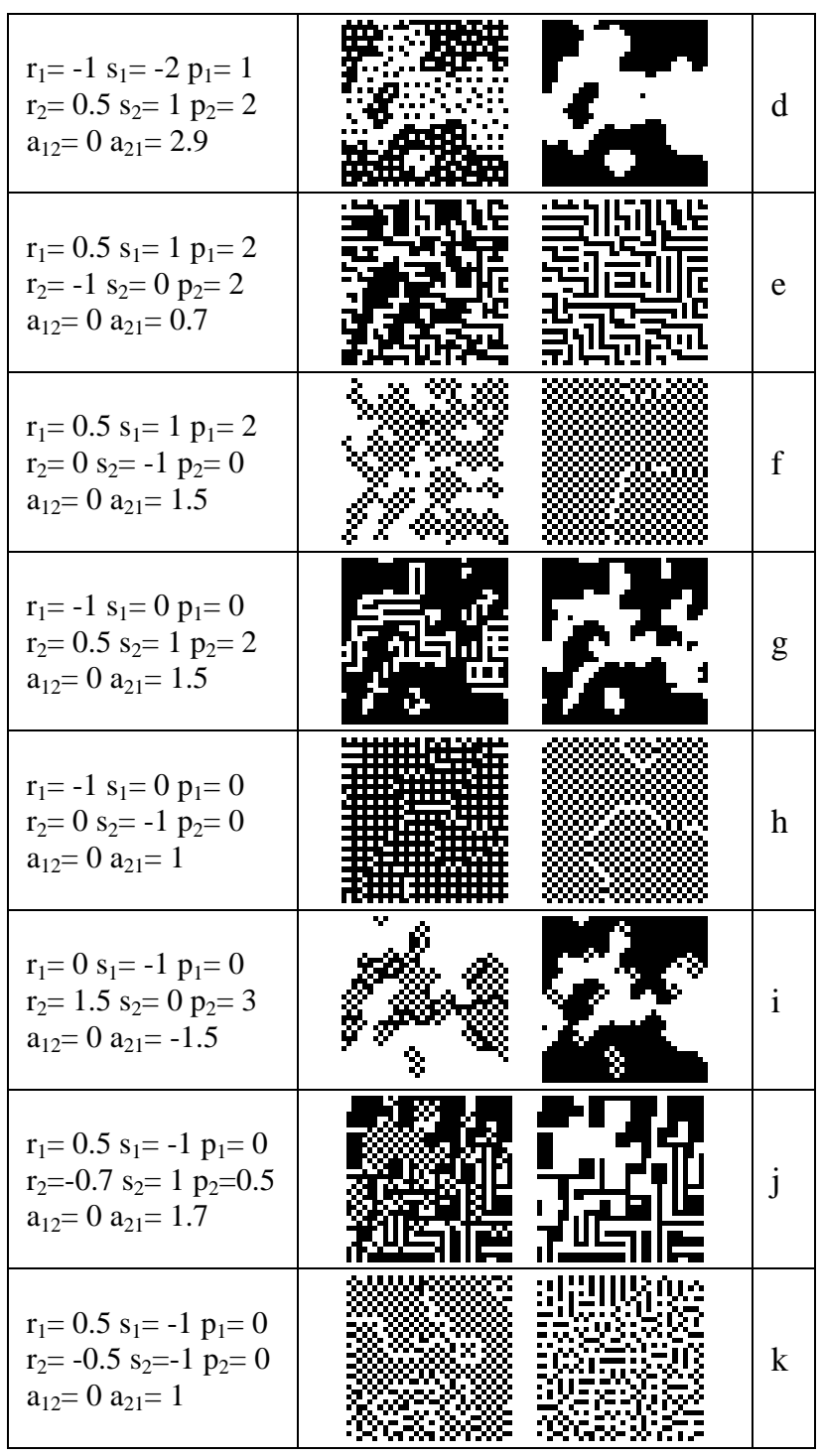

Fig.6. Some interesting coupled pattern results

In another set of measurements it is demonstrated how mutually coupled layers can generate exotic behavior, as it can be seen on Fig. 7. For example: (a) Turing-like patterns with checkerboard regions, (b) boundary snakes, and (c) wide streak regions by combining two stripe patterns. In these experiments the time constant of the first layer usually plays a significant role influencing both the transient dynamics and the steady-state properties of the network.

Some observed phenomena for different time constant settings are as follows:

- “integrated patterns”, e.g. smoothly changing number of checkers in patches (d)

- “competing patterns", e.g. change from patchcheckers to stripes (e)

- “pattern-waves”, e.g. traveling patterns with a changing width of the pattern front (f) 


\begin{tabular}{|c|c|c|}
\hline Parameters & Output of the first layer & \\
\hline $\begin{array}{l}\mathrm{r}_{1}=0 \mathrm{~s}_{1}=-1 \mathrm{p}_{1}=0 \\
\mathrm{r}_{2}=0 \mathrm{~s}_{2}=-1 \mathrm{p}_{2}=0 \\
\mathrm{a}_{12}=1 \mathrm{a}_{21}=-2\end{array}$ & 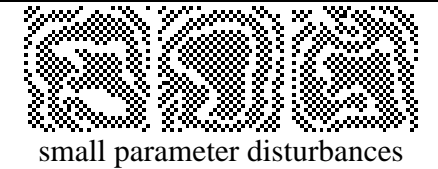 & $\mathrm{a}$ \\
\hline $\begin{array}{l}\mathrm{r}_{1}=-0.9 \mathrm{~s}_{1}=2 \mathrm{p}_{1}=-2 \\
\mathrm{r}_{2}=0.5 \mathrm{~s}_{2}=-1 \mathrm{p}_{2}=0.5 \\
\mathrm{a}_{12}=0.5 \mathrm{a}_{21}=0.5\end{array}$ & 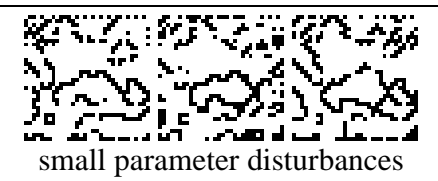 & $\mathrm{b}$ \\
\hline $\begin{array}{l}\mathrm{r}_{1}=-1 \mathrm{~s}_{1}=0 \mathrm{p}_{1}=0 \\
\mathrm{r}_{2}=-2 \mathrm{~s}_{2}=0 \mathrm{p}_{2}=0 \\
\mathrm{a}_{12}=-1 \mathrm{a}_{21}=2\end{array}$ & 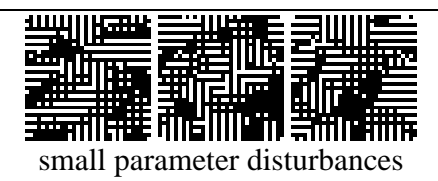 & C \\
\hline $\begin{array}{l}\mathrm{r}_{1}=-0.5 \mathrm{~s}_{1}=-1 \mathrm{p}_{1}=0 \\
\mathrm{r}_{2}=0.5 \mathrm{~s}_{2}=1 \mathrm{p}_{2}=2 \\
\mathrm{a}_{12}=3 \mathrm{a}_{21}=2\end{array}$ & $\underset{\tau=5}{\tau=9} \quad \tau=15$ & $\mathrm{~d}$ \\
\hline $\begin{array}{l}\mathrm{r}_{1}=1 \mathrm{~s}_{1}=0 \mathrm{p}_{1}=0.8 \\
\mathrm{r}_{2}=-1 \mathrm{~s}_{2}=0 \mathrm{p}_{2}=2 \\
\mathrm{a}_{12}=0.1 \mathrm{a}_{21}=1.8\end{array}$ & 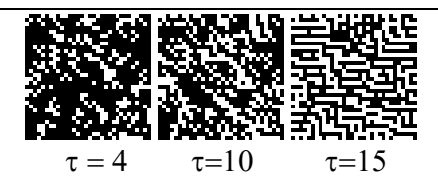 & e \\
\hline $\begin{array}{l}\mathrm{r}_{1}=0 \mathrm{~s}_{1}=-1 \mathrm{p}_{1}=0.1 \\
\mathrm{r}_{2}=0 \mathrm{~s}_{2}=-1 \mathrm{p}_{2}=0 \\
\mathrm{a}_{12}=1 \mathrm{a}_{21}=-2\end{array}$ & 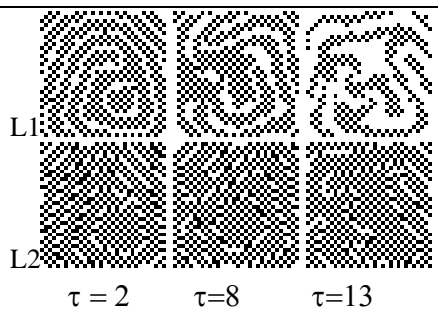 & $\mathrm{f}$ \\
\hline
\end{tabular}

Fig.7. Mutually coupled pattern formation examples

The resolution of the chip (32 by 32) doesn't make possible to properly investigate larger region and slower pattern formations. In some of these settings the results are also heavily influenced by boundary effects. In many of the cases the generated output becomes very much like a random image.

\section{CONCLUSIONS}

We have investigated the pattern formation properties of a complex cell CNN-UM chip showing a rich set of examples for the basic motifs and also for interacting patterns on a two-layer architecture. The measurement results clearly indicate that there are pattern classes that can be reliably generated, controlled and modified on CNN type architectures implemented in mixed-signal VLSI chips. This strongly motivates further research in the area of the constructive use of pattern formation dynamics and calls for new application oriented investigations similar to those found in the works of K. R. Crounse ([11], [12]).

\section{REFERENCES}

[1] I. Petrás, Cs. Rekeczky, T. Roska, R. Carmona, F. JimenezGarrido, and A. Rodriguez-Vazquez "Exploration of SpatialTemporal Dynamic Phenomena in a 32 ' 32-Cells Stored Program 2-Layer CNN Universal Machine Chip Prototype," Journal of Circuits, Systems, and Computers, Vol 12(6), 2003

[2] D. Bálya, I. Petrás, T. Roska, R. Carmona, A. Rodríguez Vázquez: "Implementing the Multi-Layer Retinal Model on the Complex-Cell CNN-UM Chip Prototype", International Journal of Bifurcation and Chaos (IJBC), Vol. 14(2), 2004

[3] R. Carmona, F. Jiménez-Garrido, R. Domínguez-Castro, S. Espejo, T. Roska, Cs. Rekeczky, I. Petrás and Á. RodríguezVázquez, "A Bio-inspired two-layer Mixed-signal Flexible Programmable Chip for Early Vision”, IEEE Transactions on Neural Networks, Vol. 14(5), pp.1313-1336, 2003

[4] L.O. Chua and T. Roska, Cellular Neural Networks and Visual Computing, Cambridge University Press, Cambridge, 2001.

[5] G. Liñan, S. Espejo, Domínguez-Castro R., Roca E. and Rodríguez-Vázquez A. "The CNNUC3: An Analog I/O 64x64 CNN Universal Machine Chip Prototype with 7-Bit Analog Accuracy”, Proc. of IEEE CNNA'2000, Catania, pp. 201-206, 2000

[6] S. Espejo, R. Carmona, Domínguez-Castro R. and Rodríguez-Vázquez A. "A VLSI Oriented Continuous-Time CNN Model," International Journal of Circuit Theory and Applications, Vol. 24(3), pp. 341-356, 1996

[7] A. Zarándy, Cs. Rekeczky, P. Földesy, and I. Szatmári: “The New Framework of Applications - The Aladdin System”, J. of Circuits, Systems, and Computers (JCSC), Vol 12(6), 2003

[8] P. Thiran, K.R. Crounse, L.O. Chua, M. Hasler, "Pattern formation properties of autonomous Cellular neural Networks", IEEE Trans. Circuits Systems I, pp. 757-774, 1995

[9] K.R. Crounse, L.O. Chua, P. Thiran and G. Setti: "Characterization and dynamics of pattern formation in Cellular Neural Networks", Intl' Journal of Bifurcation and Chaos, Vol. 6(9), pp.1703-1724, 1996

[10] Cs. Rekeczky, T. Serrano, T. Roska and Á. RodríguezVázquez "A stored program 2nd order / 3-layer complex cell CNN-UM”, Proc. of IEEE CNNA’2000, pp. 213-218, 2000

[11] K. R. Crounse, T. Roska, L.O. Chua "Image Halftoning with Cellular Neural Networks", IEEE Trans. Circuits and Systems II, Vol. 40, pp. 267-283, 1993

[12] K. R. Crounse, L.O. Chua "Methods for Image Processing and Pattern Formation in Cellular Neural Networks: A tutorial", IEEE Trans. Circuits Systems I, pp. 583-601, 1995 